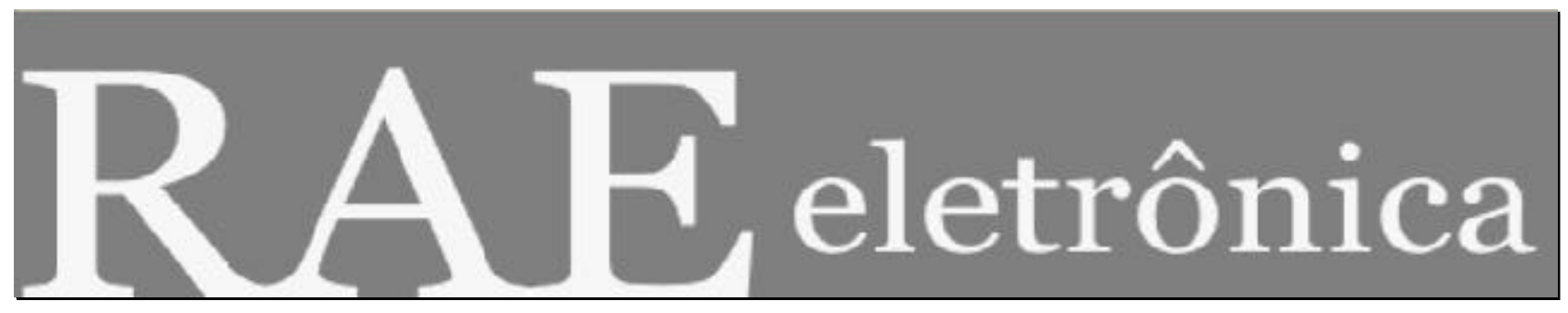

\title{
IMPACTO DE TREINAMENTO NO TRABALHO VIA INTERNET
}

Por:

Thaís Zerbini, Faculdade Millenniun de Brasília e UNB

Gardênia Abbad, UNB

RAE-eletrônica, v. 4, n. 2, Art. 16, jul./dez. 2005

http://www.rae.com.br/eletronica/index.cfm?FuseAction=Artigo \&ID=3575\&Secao=ARTIGOS\&Volu $\mathrm{me}=4 \&$ Numero $=2 \& A n o=2005$

(C) Copyright, 2005, RAE-eletrônica. Todos os direitos, inclusive de tradução, são reservados. É permitido citar parte de artigos sem autorização prévia desde que seja identificada a fonte. A reprodução total de artigos é proibida. Os artigos só devem ser usados para uso pessoal e nãocomercial. Em caso de dúvidas, consulte a redação: raeredacao@fgvsp.br.

A RAE-eletrônica é a revista on-line da FGV-EAESP, totalmente aberta e criada com o objetivo de agilizar a veiculação de trabalhos inéditos. Lançada em janeiro de 2002, com perfil acadêmico, é dedicada a professores, pesquisadores e estudantes. Para mais informações consulte o site www.rae.com.br/eletronica.

RAE-eletrônica ISSN 1676-5648

(C2005 Fundação Getulio Vargas - Escola de Administração de Empresas de São Paulo.

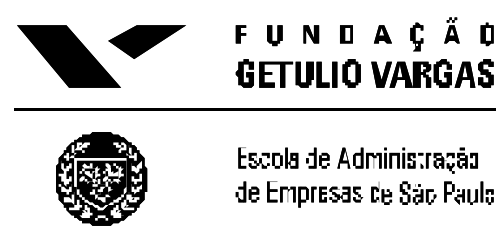




\section{RESUMO}

Esta pesquisa teve como objetivo testar um modelo reduzido de avaliação de Impacto de Treinamento no Trabalho. Foram realizados dois estudos: (1) Construção e validação estatística de cinco instrumentos de medida: Escalas de Estratégias de Aprendizagem, Reação aos Procedimentos Instrucionais; Reação ao Desempenho do Tutor, Falta de Suporte à Transferência e Impacto em Profundidade; (2) Análise do relacionamento entre as variáveis preditoras referentes às Características da Clientela, Reações ao Treinamento, Falta de Suporte à Transferência e à variável-critério Impacto do Treinamento no Trabalho. As respostas dos participantes às escalas foram submetidas a análises exploratórias dos componentes principais, análise fatorial, de consistência interna, análises de regressão múltipla padrão e stepwise. No Estudo 1, todos os instrumentos apresentaram índices psicométricos satisfatórios. No Estudo 2, as variáveis explicativas de Impacto do Treinamento no Trabalho foram: Falta de Suporte, Estratégias Cognitivas e Comportamentais, Elaboração de um Plano de Negócio e Reação aos Procedimentos. Neste artigo, são discutidas algumas implicações desses resultados.

\section{ABSTRACT}

This research has had as a goal to test a reduced model of evaluation of Work Training Impact. Two studies have been done: (1) Construction and Validating Statistics of five measure instruments: Scales of Learning Strategies, Reaction to Instructional Proceedings and to the Tutor Performance, Lack of Support to Transference and Deep Impact; (2) To analyse the relationship between the predicts variables referred to the Customer's Characteristics, Reactions to Training, Lack of Support to Transference and the variable criteria Work Training Impact. The attendants answers to the scales have been submitted to exploiting analyse, of the main components, factorial analyse, of internal consistence, analyses of patterned multiple regression and stepwise. In the Study 1, all the instruments showed satisfactory psychometrics results. In the Study 2, the explainable variables of Work Training Impact were: Lack of Support, Cognitive and Behaviourist Strategies, Elaboration of a Business Plan, Reaction to the Proceedings. Some of the implications of theses results are discussed in this paper.

\section{PALAVRAS-CHAVE}

Avaliação de treinamento à distância, estratégias de aprendizagem, reações, suporte à transferência e impacto do treinamento no trabalho.

\section{KEYWORDS}

Evaluation of distance training, learning strategies, reactions, support to transference and work training impact. 


\section{INTRODUÇÃO}

Atualmente, as estratégias de gestão de aprendizagem mais usadas pelas organizações são os programas formais de treinamento, desenvolvimento e educação (TD\&E). Porém, apesar dos altos investimentos, muitas organizações ainda utilizam sistemas de treinamentos inadequados à sua realidade, em virtude da ausência de um processo sistemático de levantamento de necessidades e de avaliação dos resultados obtidos por meio de treinamentos. É essencial o desenvolvimento de estudos que visem produzir conhecimentos na área de avaliação, desenvolver soluções técnicas para a busca de sistemas que ofereçam maior produtividade, lucratividade e competitividade para as empresas e para os indivíduos. O desenvolvimento e utilização das novas tecnologias de informação e comunicação (NTICs) em treinamentos corporativos e abertos estão se tornando essenciais para facilitar a aquisição e atualização de conhecimentos dos indivíduos.

Borges-Andrade (2002, p. 31) faz uma observação às rápidas mudanças tecnológicas, econômicas e sociais que estão ocorrendo no ambiente do trabalho e das organizações. Segundo o autor, a busca pelo desenvolvimento de competências pessoais deixou de ser uma decisão pessoal e passou a ser estratégia organizacional, e treinamento, por sua vez, passa a ser visto pelos indivíduos como oportunidade de melhorar o desempenho, não so mente no cargo atual como também em cargos futuros e em outras organizações. $O$ autor concluiu que os conceitos de TD\&E podem estar apresentando um enfraquecimento nas fronteiras que os separavam há menos de duas décadas, já que programas de desenvolvimento e educação, assim como treinamentos, estão sendo usados pelas empresas e organizações como estratégia organizacional. Verifica-se que, apesar de diferentes conceitualmente, as ações de TD\&E, instrução e informação, quando usadas adequadamente e em conjunto, visam promover e acelerar o processo de aprendizagem nas organizações. As diferenças entre elas, na verdade, são as vantagens específicas que cada ação pode oferecer para a organização.

Conhecida a tendência do enfraquecimento das fronteiras existentes entre os conceitos de $\mathrm{TD} \& \mathrm{E}$, o treinamento a distância $(\mathrm{TaD})$ pode ser entendido como um conjunto de ações educacionais, sistematicamente planejadas, desenvolvidas na maior parte do tempo no contexto de flexibilidade espacial e temporal entre professor e aluno, de sincronidade e assincronidade, de interação e interatividade e de abertura dos espaços físicos, utilizando-se de meios tecnológicos que facilitam o aperfeiçoamento e a aquisição de conhecimentos, habilidades e atitudes (CHAs) por meio do autogerenciamento da aprendizagem do indivíduo. Os CHAs adquiridos podem ser aplicados no trabalho atual ou futuro, em empresas diferentes, e, no caso de usuários autônomos, aplicados nas diversas atividades profissionais desenvolvidas pelo indivíduo.

O TaD pode facilitar o desenvolvimento de carreiras dentro e fora das organizações por meio de currículos e trilhas educacionais voltados para educação continuada. Em função disso, as características que distinguem o treinamento a distância de treinamentos presenciais deveriam estar produzindo mudanças nos modelos de avaliação de treinamentos a distância e em seus instrumentos de coleta de dados. A proposta desta pesquisa, portanto, foi desenvolver um modelo de avaliação de treinamento a distância com variáveis específicas de planejamento, execução e estratégias de aprendizagem utilizados em cursos a distância. 


\section{REVISÃO DE LITERATURA: AVALIAÇÃO DE TREINAMENTO}

Borges-Andrade e Abbad (1996, p. 112) concluíram que o uso da abordagem sistêmica como referencial teórico é predominante nas pesquisas analisadas. Os elementos que compõem o sistema de treinamento são: avaliação de necessidades de treinamento, planejamento e execução de treinamento e avaliação de treinamento, sendo que esses mantêm entre si trocas constantes de informações e resultados. A avaliação de treinamento é um elemento essencial, já que é responsável pelo fornecimento de retroalimentações que asseguram o aperfeiçoamento constante do sistema de treinamento.

Entre os modelos clássicos de avaliação estão os propostos por Kirkpatrick (1976, p. 18.1) e Hamblin (1978). Kirkpatrick (1976, p. 18.1) propôs um modelo em quatro níveis de avaliação: reação, aprendizagem, comportamento e resultados. Hamblin (1978) adaptou o modelo, desdobrando o quarto nível de avaliação em dois, e propôs cinco níveis de avaliação de treinamento: reação, aprendizagem, comportamento no cargo, mudança organizacional e valor final. Kirkpatrick (1976, p. 18.1) sugere em seu modelo que os níveis de avaliação são seqüenciais, lineares e fortemente correlacionados entre si. Os modelos clássicos não têm apoio empírico da área de avaliação. Pesquisas mais recentes mostraram que a relação hierárquica e positiva entre os níveis de avaliação não é uma realidade. Nos resultados encontrados por Abbad (1999), aprendizagem relaciona-se fracamente com reações e não tem relacionamento significativo com impacto.

Os modelos tradicionais de avaliação incluem em seus componentes apenas variáveis de resultados de treinamento. O Modelo de Avaliação Integrado e Somativo - MAIS - de Borges-Andrade (1982, p. 29) acrescenta variáveis do ambiente e de processo na avaliação de resultados de treinamento, e Abbad (1999) desenvolveu o Modelo Integrado de Avaliação do Impacto do Treinamento no Trabalho (IMPACT). Este modelo avalia a relação entre os níveis de avaliação reação, aprendizagem e impacto e investiga diversas variáveis preditivas de eficiência e eficácia do treinamento.

A maioria dos estudos identificados na área de avaliação de treinamento a distância relata características conceituais de educação a distância e não apresenta questões ou resultados de pesquisas empíricas. Apenas nove trabalhos entre mais de cem artigos e teses foram selecionados descreviam pesquisas empíricas sistemáticas. Em todos eles foram descritos e aplicados questionários para avaliar apenas o nível de reações, com exceção de Warr e Bunce (1995, p. 347), que desenvolveram um estudo para examinar o impacto de certas características de treinandos nos resultados de um programa de aprendizagem aberta.

Cheung (1998, p. 23) desenvolveu um questionário de avaliação da qualidade de ensino em cursos a distância composto por quatro dimensões: desenvolvimento do estudante, avaliação, materiais de aprendizagem e componente face a face. O objetivo foi desenvolver e validar uma escala de efetividade do ensino em cursos de educação a distância (EaD). Dean e Webster (2000, p. 344) desenvolveram e testaram um instrumento para avaliar diversos recursos instrucionais utilizados em cursos a distância, em especial as simulações. As análises fatoriais apontaram três dimensões: aspectos do programa de computador, motivação para estudar e capacidade de transferir o conhecimento para o local de trabalho. 
Outros autores desenvolveram instrumentos de medida e avaliações no nível de reação, entretanto não apresentaram resultados estatísticos dignos de nota. Salas e Cannon-Bowers (2001, p. 471) falam, em sua revisão da área de TD\&E, que há poucos estudos na área de treinamento a distância e alguns autores apenas "começaram arranhar a superfície desse tópico" (bidem., p. 483). Abbad, Pantoja e Pilati (2003, p. 181) afirmam que, para que fosse possível a ocorrência de maiores avanços na área, seria preciso analisar cuidadosamente o $\mathrm{TaD}$, verificando as variáveis explicativas de seus resultados. Partindo dessa demanda e dos (poucos) achados da área, foram detectadas lacunas e falhas na construção de instrumentos e ausência de modelos de avaliação para cursos a distância. Conhecendo a importância da avaliação de treinamentos, é proposto neste estudo um modelo reduzido de avaliação de treinamentos a distância, adaptado do modelo de avaliação de treinamento presencial desenvolvido a partir do modelo de Abbad (1999), com o intuito de analisar o relacionamento entre as variáveis Características Individuais, Reação aos Procedimentos Instrucionais, Reação ao Desempenho do Tutor, Falta de Suporte à Transferência e Impacto do Treinamento.

\section{Características individuais}

As variáveis referentes ao componente Características Individuais apresentam menor poder de explicação de impacto se comparadas às variáveis relacionadas às da categoria "Suporte e Clima para Transferência". Em cursos a distância pela Internet, as características individuais podem ter maior influência nos efeitos do treinamento por se tratar de uma aprendizagem que depende muito mais do esforço do próprio indivíduo (autogestão da aprendizagem) do que dos recursos instrucionais em si; o indivíduo provavelmente utilizará diferentes estratégias usadas nos cursos presenciais em função dos novos procedimentos instrucionais específicos de treinamentos baseados na Web (TBWs). O estudo das estratégias de aprendizagem pode ser útil para auxiliar o planejamento instrucional de cursos a distância ao verificar as estratégias e estilos mais utilizados pelos indivíduos, bem como ao analisar para quais pessoas são mais adequados determinados procedimentos oferecidos.

Segundo Warr e Allan (1998, p. 83), existem três conceitos que eventualmente podem gerar dúvidas: habilidades/hábitos de estudo, estilos de aprendizagem e estratégias de aprendizagem. Hábitos de estudo envolvem os procedimentos utilizados pelo aluno para integrar os melhores aspectos contextuais e maneiras de estudo com o objetivo de melhorar a aquisição e retenção da aprendizage m. Exemplo de hábitos de estudo são habilidades de leitura, gerenciamento do tempo, uso efetivo de biblioteca, procedimentos de revisão e fazer anotações, entre outros. Estilos de aprendizagem são as preferências do estudante referentes aos aspectos contextuais e às maneiras de estudo. Compreendem a preferência dos indivíduos por determinadas estratégias de aprendizagem e por aspectos do contexto em que esta ocorre. Exemplo de estilos de aprendizagem: nível de ruído e temperatura do ambiente ao estudar, horário de preferência de estudo, forma de estudo (individual, em grupo), maneira de adquirir e processar as informações (pedir ajuda a colegas, consultar o material). Estratégias de aprendizagem são procedimentos utilizados pelos indivíduos durante as atividades de aprendizagem para serem bemsucedidos. As estratégias variam mais do que os estilos, já que podem ter a influência das preferências gerais do indivíduo. Além disso, os autores dizem que as estratégias podem ser modificadas em treinamento com o intuito de aumentar a efetividade da aprendizagem em uma atividade ou ambiente específico. Isso significa dizer que não existem estratégias melhores, e sim estratégias mais adequadas ao tipo de atividade a ser aprendida. 
Warr e Allan (1998, p. 83) atribuem a mesma importância ao estudo de estilos e estratégias de aprendizagem, já que informações sobre as preferências do indivíduo podem ser úteis para adaptar os procedimentos utilizados no evento instrucional. Já informações sobre estratégias utilizadas podem identificar pontos a serem trabalhados no indivíduo para melhorar seu aproveitamento durante o curso sem necessariamente modificar os procedimentos.

Conhecida a discussão sobre a dificuldade dos alunos em se adaptarem às novas tecnologias de ensino, tais como o uso de chats, fóruns, troca de e-mails e como salvar arquivos, entre outros, informações sobre as estratégias usadas em cursos oferecidos pela Internet serão de extrema utilidade para verificar, empiricamente, se as ferramentas disponibilizadas estão sendo usadas de maneira efetiva pelos alunos ou se será necessário um pré-treinamento para ensinar-lhes estratégias adequadas a fim de aumentar o aproveitamento das atividades oferecidas.

Warr e Allan (1998, p. 83) propuseram uma taxonomia de estratégias de aprendizagem para treinamento profissional em adultos. Os autores consultaram diversos inventários de estratégias de aprendizagem utilizados nos contextos escolar e universitário para tornar possível a construção da taxonomia em questão. A taxonomia é formada por três categorias, as quais compreendem diferentes níveis de análise; a saber:

- Estratégias de Aprendizagem Cognitiva são compostas pelas estratégias: 1) Repetição repetição mental da informação na forma em que foi apresentada; 2) Organização identificação de idéias centrais do material e criação de esquemas mentais que agrupam e relacionam elementos que foram aprendidos; e 3) Elaboração - reflexão sobre implicações e conexões possíveis entre o material aprendido e o conhecimento já existente.

- Estratégias de Aprendizagem Comportamentais agrupam as estratégias: 1) Busca de ajuda interpessoal - obtenção de auxílio de outras pessoas, como pares e professores, para tirar dúvidas sobre o material. Representa um comportamento proativo do indivíduo de solicitar ajuda ao invés de utilizar apenas as constantes informações do próprio procedimento instrucional; 2) Busca de ajuda no material escrito - obtenção de informações em documentos escritos, manuais de instruções, programas de computador e outras fontes que não envolvam contato social; e 3) Aplicação prática aprimoramento do conhecimento por meio de aplicação prática do que foi aprendido.

- Estratégias auto-regulatórias são formadas pelas estratégias: 1) Controle da emoção controle da ansiedade e prevenção de dispersões de concentração, causadas por sentimentos de ansiedade; 2) Controle da motivação - controle da motivação e da atenção, apesar de existência de um interesse limitado na tarefa a ser aprendida; e 3) Monitoramento da compreensão - avaliação do processo de aquisição de aprendizagem e modificação do comportamento do indivíduo quando necessário.

Warr e Downing (2000, p. 311) construíram e validaram um instrumento de 45 itens distribuídos em 8 fatores com bons índices de consistência interna. Os autores sugerem a aplicação de pré e pós-teste para avaliar as mudanças ocorridas nas estratégias de aprendizagem do começo até o fim do treinamento. Essa sugestão pode facilitar a diferenciação entre o que pode ser considerado resultante do processo do evento instrucional e o que pode ser atribuído às características disposicionais do indivíduo. Para Borges-Andrade (1982, p. 29), variáveis de processo referem-se a tudo o que o aluno 
sente, diz, pensa ou faz à medida que os procedimentos são implementados. Nesse sentido, se as estratégias cognitivas, motivações e outras características disposicionais são modificadas durante o evento instrucional, elas podem ser analisadas como variáveis de processo, na concepção de BorgesAndrade (1982, p. 29).

\section{Reações ao treinamento, suporte à transferência e impacto de treinamento no trabalho}

Quanto ao componente "Reações", Abbad, Gama e Borges-Andrade (2000, p. 25) obtiveram duas escalas confiáveis: reações ao treinamento e reações ao desempenho do instrutor. Os autores concluíram que aprendizagem é a única variável que não está significativamente relacionada com todas as demais e que as medidas de reações são fortemente relacionadas com impacto. Walker (1998, p. 270) mediu a satisfação dos alunos em relação aos exemplos fornecidos durante o curso, aos materiais usados, à relação entre o conteúdo do curso e sua aplicação prática no trabalho. Além disso, avaliou a satisfação dos alunos referentes ao desempenho do instrutor, segundo os aspectos conhecimento do conteúdo e incentivo nas participações.

As variáveis de "Suporte e Clima para Transferência" vêm sendo apontadas, tanto em pesquisas nacionais quanto em estrangeiras, como as mais importantes preditoras de impacto do treinamento no trabalho a longo prazo (Roullier e Goldstein, 1993, p. 377; Salas e Cannon-Bowers, 2001, p. 471; Abbad, Pantoja e Pilati, 2003, p. 181). Segundo Abbad, Pantoja e Pilati (2003, p. 181), não há estudos sobre quais variáveis de suporte são necessárias à transferência de competências aprendidas em $\mathrm{TaD}$.

Segundo Abbad (1999), o impacto do treinamento no trabalho corresponde ao terceiro nível de avaliação proposto por Kirkpatrick (1976, p. 18.1) e Hamblin (1978), podendo ser entendido como o efeito do treinamento no desempenho do indivíduo ao retornar para o trabalho. Impacto de treinamento no trabalho é um conceito muito utilizado em pesquisas que envolvem T\&D no Brasil. Segundo Abbad (1999), para observar o desempenho do indivíduo no trabalho, ou seja, a aplicação do aprendido no ambiente de transferência, é preciso mais do que saber fazer (habilidades). O indivíduo também precisa querer fazer (motivação) a tarefa e ter condições do ambiente para tal. Impacto do treinamento compreende noções de desempenho e motivação. A autora sugere que, para medir os resultados de um treinamento, deve-se avaliar o desempenho, a motivação e as atitudes da clientela depois do término do treinamento. $\mathrm{O}$ impacto pode ser medido em profundidade ou largura. Impacto em profundidade mede os efeitos do treinamento que estão relacionados aos conteúdos ensinados nos programas de treinamentos. Impacto em largura ou amplitude mede os efeitos do treinamento referentes a desempenhos gerais esperados pela organização. O conceito de Impacto em Profundidade foi cunhado por Hamblin para distinguir efeitos diretos e específicos de um evento instrucional de outros, mais gerais e não extraídos diretamente dos objetivos de ensino, por ele denominado Impacto em Amplitude. Existem auto e heteroavaliações. A primeira é feita pelos próprios treinandos e a segunda, por supervisores, colegas ou clientes. $\mathrm{O}$ ideal é utilizar os dois tipos de avaliação no mesmo treinamento, porém há um número bem menor de pesquisadores que estudaram impacto em profundidade como variável-critério. Nesta pesquisa, a variável-critério será impacto em profundidade. A seguir são relatados os objetivos da pesquisa. 


\title{
OBJETIVOS DA PESQUISA
}

\begin{abstract}
Abbad, Pantoja e Pilati (2003, p. 181) verificaram a necessidade de mais pesquisas para criação e/ou validação de modelos multivariados de avaliação de resultados de treinamentos presenciais e a distância. A revisão de literatura revela também a ausência de modelos de avaliação para cursos a distância via Internet. Pretende-se estudar, em caráter exploratório, qual a direção e a magnitude da contribuição de variáveis referentes aos indivíduos, aos resultados de treinamento e às condições do ambiente na explicação de impacto de um treinamento a distância, via Internet, no trabalho. Para tanto, foi necessário construir e validar instrumentos de medida de estratégias de aprendizagem, reação ao curso e ao tutor, falta de suporte à transferência e impacto (em profundidade) do treinamento do trabalho, a fim de que análises sistemáticas do relacionamento entre essas variáveis pudessem ser realizadas.
\end{abstract}

Espera-se que, em cursos a distância mediados pela Internet, características individuais exerçam maior influência sobre impacto do treinamento no trabalho do que em situações de cursos presenciais. Espera-se também que o indivíduo utilize estratégias diferentes daquelas usadas nos cursos presenciais em função dos novos procedimentos instrucionais específicos de treinamentos baseados na Web $(T B W s)$. Em situação de curso a distância, a satisfação do participante com o treinamento provavelmente contribui mais fortemente para a explicação de impacto do treinamento no trabalho do que em treinamentos presenciais, visto que a escolha dos recursos e meios instrucionais em $T B W \mathrm{~s}$ demandam um planejamento prévio mais detalhado e específico do que ocorre em cursos tradicionais. Resultados de pesquisas sobre treinamentos presenciais em ambientes organizacionais analisadas na revisão de literatura mostraram que a falta de suporte à transferência influencia negativamente o impacto do treinamento no trabalho e que suporte é uma das variáveis que mais explicam o impacto. Neste estudo, espera-se que os resultados sejam similares a esses.

O presente trabalho compreende dois estudos, orientados pelos seguintes objetivos: 1) Construção e validação estatística dos seguintes instrumentos de medida: Escalas de Estratégias de Aprendizagem, Escalas de Reação aos Procedimentos Instrucionais, Escalas de Reação ao Desempenho do Tutor, Escala de Falta de Suporte à Transferência e Escala de Avaliação de Impacto em Profundidade do curso; 2) Análise do relacionamento entre as variáveis preditoras referentes às Características da Clientela, Reações ao Treinamento, Falta de Suporte à Transferência e a variávelcritério Impacto do Treinamento IPGN no Trabalho.

\section{MODELO DE INVESTIGAÇÃO}

O modelo de investigação adotado neste estudo é derivado dos modelos MAIS (Borges-Andrade, 1982, p. 29) e Impact (Abbad, 1999) e possui delineamento multivariado. O modelo é composto por quatro componentes: (1) Características da Clientela, (2) Reações, (3) Falta de Suporte à Transferência e (4) Impacto do Treinamento no Trabalho, como pode ser observado na Figura 1. 
Figura 1 - Modelo geral de avaliação do impacto de treinamento via Web no trabalho

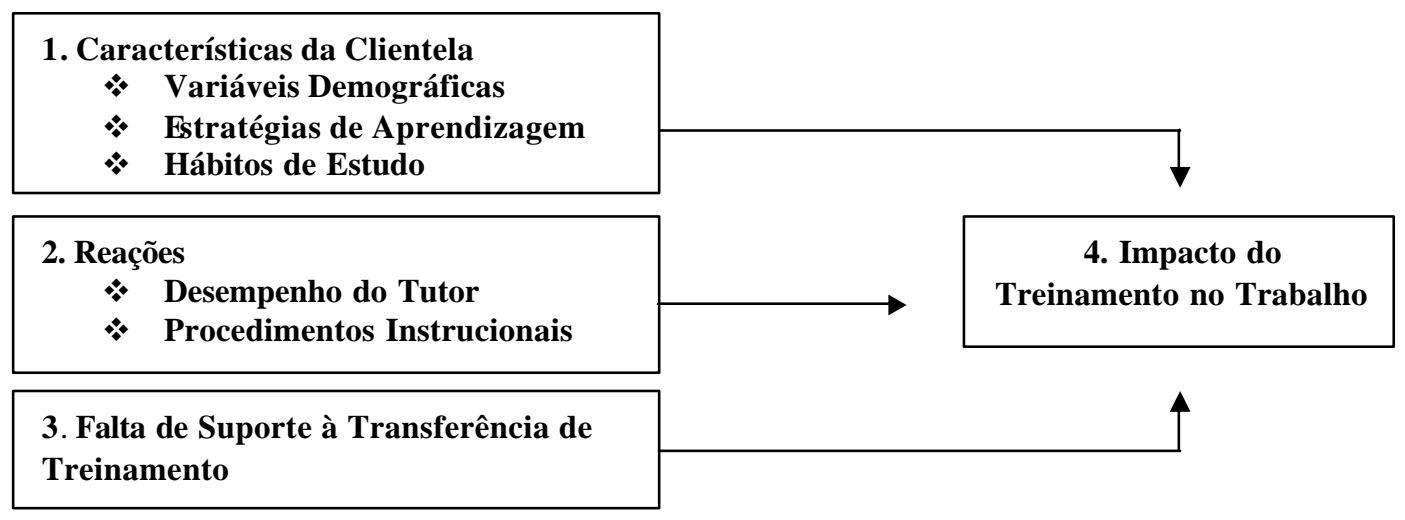

O componente Características da Clientela é composto por Dados Demográficos dos participantes (sexo, idade, região geográfica, nível de escolaridade, experiência na Internet, área de negócios de interesse, se está na situação de proprietário de empresa, participações anteriores em cursos a distância, ocupação atual) e Estratégias de Aprendizagem e Hábitos de Estudo.

Estratégias de Aprendizagem envolve capacidades cognitivas e habilidades comportamentais utilizadas pelo aprendiz para controlar os próprios processos psicológicos de aprendizagem, como atenção, aquisição, memorização e transferência. A definição dessa variável, neste estudo, não inclui as estratégias de autocontrole emocional propostas por Warr e Allan (1998, p. 83) e Warr e Downing (2000, p. 311). Hábitos de Estudo, refere-se às preferências dos indivíduos por determinadas estratégias de aprendizagem e por aspectos do contexto de estudo que o levam a escolher horário e formas de estudo, maneiras de adquirir e processar as informações, horas de estudos na Internet, horas de estudo fora da Internet, horário preferido de estudo e quantidade de leitura do material do curso.

Reações ao Treinamento refere-se à satisfação dos participantes com características instrucionais do curso, como qualidade dos objetivos de ensino, conteúdos, sequiência, avaliações de aprendizagem, estratégias e meios, assim como à qualidade das ferramentas da Web. Refere-se, ainda, à percepção do treinando sobre a qualidade da interação do tutor com os alunos, domínio do conteúdo e uso de estratégias de ensino.

Falta de Suporte à Trans ferência avalia a opinião dos participantes acerca do nível com que variáveis do contexto familiar, social e/ou governamental podem prejudicar o negócio ou a aplicação das habilidades aprendidas no curso Iniciando um Pequeno Grande Negócio (IPGN).

Impacto do Treinamento no Trabalho, quarto componente do modelo, refere-se aos efeitos específicos do treinamento sobre o desempenho do participante em atividades diretamente relacionadas aos CHAs desenvolvidos no treinamento. Trata-se de uma medida de avaliação de Impacto em Profundidade. 


\section{MÉTODO}

\section{Características da organização, do curso e das amostras}

A presente pesquisa foi realizada no período de janeiro a dezembro de 2002 e consistiu da avaliação do curso IPGN, ofertado pelo Sebrae Nacional (Serviço de Apoio às Micro e Pequenas Empresas). O curso é gratuito, realizado totalmente a distância via Internet com tutoria ativa, tem a duração prevista de 40 horas e visa ensinar, passo a passo, a elaboração de um plano de negócios. O total de inscritos no IPGN foi de 21.920 alunos, divididos em 110 turmas de, aproximadamente, 200 alunos cada uma. O Quadro 1 apresenta uma comparação entre os perfis da população de inscritos no curso IPGN e as amostras dos estudos/objetivos 1 e 2 (validação de instrumentos e análises de regressão, respectivamente).

\section{Quadro 1 - Comparação entre os perfis de inscritos e amostras}

\begin{tabular}{|c|c|c|c|c|c|c|c|c|}
\hline \multicolumn{9}{|c|}{ Comparação entre os perfis de participantes } \\
\hline \multirow[t]{2}{*}{ Variáveis } & \multirow[t]{2}{*}{$\begin{array}{l}\text { População } \\
\text { de Inscritos }\end{array}$} & \multicolumn{5}{|c|}{ Estudo 1 - Validação de Instrumentos } & \multicolumn{2}{|c|}{$\begin{array}{c}\text { Estudo } 2 \text { - Análises } \\
\text { de Regressão }\end{array}$} \\
\hline & & EEA* & ERPI* & ERDT* & ESIPGN* & EIPGN* & Modelo 1 & Modelo 2 \\
\hline Concluinte & $52,9 \%$ & $86,7 \%$ & $85,3 \%$ & $86,6 \%$ & $91,4 \%$ & $89,1 \%$ & $99 \%$ & $95,5 \%$ \\
\hline Sexo & Masc. & Masc. & Masc. & Masc. & Masc. & Masc. & Masc. & Masc. \\
\hline Idade & $33 / 9,29$ & $34 / 9,77$ & $34 / 10$ & $34 / 9,81$ & $35 / 10$ & $34 / 10$ & $34 / 10$ & $35 / 10$ \\
\hline \multicolumn{9}{|l|}{ Média/ DP } \\
\hline Região & Sudeste & Sudeste & Sudeste & Sudeste & Sudeste & Sudeste & Sudeste & Sudeste \\
\hline
\end{tabular}

As amostras são muito semelhantes à população de inscritos no curso no que diz respeito ao sexo (masculino), região de residência (Sudeste) e média de idade (34 anos). Em todas as amostras foi pouco freqüente o uso das ferramentas da $W e b$.

\section{Instrumentos de medida - construção e validação estatística}

Os itens referentes a Estratégias de Aprendizagem e Hábitos de Estudo foram construídos com base nos instrumentos de Warr e Allan (1998, p. 83) e Warr e Downing (2000, p. 311). O primeiro conjunto de itens solicita ao participante que descreva o modo pelo qual ele controla os próprios processos psicológicos de aprendizagem (atenção, aquisição, memorização e transferência) e inclui dois componentes: estratégias cognitivas, como, por exemplo, resumir textos, repetir mentalmente os 
conteúdos etc., e estratégias comportamentais (busca de ajuda interpessoal para tirar dúvidas, aplicação prática dos conhecimentos adquiridos etc.). Esses dois componentes da definição de estratégias de aprendizagem assemelham-se aos propostos pelos autores anteriormente citados, porém o instrumento aplicado neste estudo excluiu os itens referentes ao componente autocontrole emocional presente nos questionários desses autores. $\mathrm{O}$ instrumento de Estratégias de Aprendizagem apresentou uma estrutura tridimensional: Busca de Ajuda Interpessoal (8 itens, alfa de 0,85); Elaboração e Aplicação Prática do Conteúdo (5 itens, alfa de 0,75); e Repetição, Organização e Ajuda do Material (7 itens, alfa de 0,78). A variância total das respostas dos participantes ao instrumento foi de, respectivamente, 12,2\%, 17\%, $5 \%$ e $12,65 \%$. A correlação entre os fatores 1 e 2 é de 0,19 ; entre os fatores 1 e 3 é de - 0,31; e entre os fatores 2 e 3 é de - 0,29.

Os itens do questionário de Reação aos Procedimentos Instrucionais foram elaborados a partir da definição do componente Procedimento do modelo MAIS (Borges-Andrade, 1982, p. 29). Foram realizadas três oficinas de trabalho, da qual participaram professores e alunos de graduação e de pósgraduação do Instituto de Psicologia da Universidade de Brasília. As oficinas de trabalho tiveram duração de 4 horas cada uma. No início dos trabalhos, os participantes receberam uma apostila contendo as definições dos componentes dos modelos de avaliação de Borges-Andrade (1982, p. 29) e Abbad (1999). Em seguida, aplicourse a técnica de "tempestade cerebral" (brainstorming) com o objetivo de levantar indicadores de procedimentos instrucionais, a partir dos quais foram redigidas as primeiras versões dos itens de Reação. O instrumento de Reação aos Procedimentos compõe-se de 19 itens, associados a uma escala de intensidade de 11 pontos $(0=$ péssimo; $10=$ excelente $)$. A validação estatística apresenta alfa de Cronbach de 0,93 e 44,26\% de variância explicada.

O instrumento de avaliação de Reação ao Desempenho do Instrutor foi construído com base na técnica dos incidentes críticos, aplicada a uma amostra de 10 (dez) tutores escolhida pelo Sebrae. A coleta dos incidentes consistiu no envio de uma mensagem aos tutores, na qual se solicitava a descrição de 5 (cinco) comportamentos observáveis que caracterizassem desempenho eficaz de um tutor e 5 (cinco) que caracterizassem comportamentos ilustrativos de desempenho ineficaz. O pedido foi enviado por e-mail e as mensagens eletrônicas de resposta eram automaticamente armazenadas em um arquivo de dados do Excel. Além dessas informações, foram analisadas 100 mensagens de ex-alunos, contidas nos chats de discussão, referentes às suas opiniões e comentários sobre a versão piloto do curso. Essas informações foram submetidas à análise qualitativa de conteúdo a fim de identificar dimensões que representassem o desempenho de um tutor em um curso a distância pela Internet. O instrumento de Reação ao Desempenho do Tutor é composto por 44 itens, com uma escala de freqüência de 11 pontos $(0=$ nunca; $10=$ sempre $)$, e apresentou alfa de Cronbach de 0,98 , com explicação de $57 \%$ da variância total das respostas.

O questionário de Falta de Suporte à Transferência do IPGN foi construído a partir de entrevistas individuais com 10 (dez) empreendedores de sucesso, nas quais foram coletados aspectos do ambiente pós-treinamento que facilitam ou inibem a abertura de um negócio no mercado. Foram realizadas entrevistas semi-estruturadas com apoio de roteiro e registro simultâneo de informações. Os indicadores levantados foram submetidos a uma análise qualitativa, com a finalidade de identificar as dimensões do construto. A Escala de Falta de Suporte à Transferência incluiu 15 itens associados a uma escala que variava de $0=$ nada a $10=$ totalmente. $\mathrm{O}$ instrumento apresentou alfa de Cronbach de 0,91 e explicação de 40,93\% da variância total das respostas.

Os itens do instrumento de Impacto do Treinamento no Trabalho foram construídos a partir da análise dos objetivos instrucionais do IPGN. Trata-se de uma medida em profundidade, que avalia 
efeitos diretos do curso sobre desempenhos específicos, estritamente relacionados aos CHAs desenvolvidos por meio do treinamento. O Impacto do Treinamento no Trabalho (em profundidade) foi composto de 24 itens vinculados à escala de freqüência de 11 pontos $(0=$ nunca a $10=$ sempre $)$. $\mathrm{O}$ alfa de Cronbach foi de 0,96 e apresentou $52,01 \%$ de variância explicada pelo fator. O instrumento incluiu mais um item, "Elaborei meu plano de negócio", por se tratar do objetivo final planejado pelo curso, porém sem obrigatoriedade de ser realizado. Este item foi respondido com opções $1=$ "sim" e $2=$ "não".

\section{Procedimentos de coleta e análise de dados}

Os instrumentos foram digitalizados e hospedados em páginas da Internet. A coleta de dados ocorreu em dois momentos distintos, ao final (último dia do curso) e após (42 a 105 dias) o término do treinamento. Os questionários foram enviados por e-mail para três grupos diferentes, conforme as respectivas datas de início e término do curso. No Momento 1, foram enviados os questionários de Dados Pessoais, Estratégias de Aprendizagem, Hábitos de Estudo, Reação aos Procedimentos Instrucionais e Reação ao Desempenho do Tutor. No Momento 2, foram aplicados os questionários de Falta de Suporte à Transferência e Impacto do Treinamento no Trabalho. O índice de retorno foi muito baixo, $24 \%$ de retorno no Momento 1 e $8,5 \%$ no Momento 2. Esse fato pode ser explicado por alguns fatores: grande porcentagem de evadidos do curso; dificuldades tecnológicas enfrentadas durante a coleta de dados por meio eletrônico; ausência de vínculo do participante com a empresa; gratuidade do curso do Sebrae; e o tamanho do intervalo entre o final do curso e a data de envio dos que stionários de impacto e suporte. As respostas dos participantes aos questionários eram automaticamente registradas em um arquivo de dados eletrônico no programa Access e, em seguida, importados pelo SPSS, versão 10.0, para que pudessem ser analisadas. Foram criados 9 arquivos de dados de modo a otimizar o aproveitamento de casos válidos.

Foram realizadas análises descritivas e exploratórias para investigar a exatidão da entrada dos dados, a presença de casos extremos, a distribuição dos casos omissos, a distribuição das variáveis e o tamanho das amostras. Para identificação dos casos extremos univariados, todas as variáveis foram transformadas em escores Z. Foram excluídas todas as respostas cujos escores padronizados eram iguais ou superiores a 3,29 ( $\mathrm{p}<0,001$, two-tailed). Os casos extremos multivariados foram identificados a partir da distância Mahalanobis $(\alpha=0,001)$ e, posteriormente, excluídos. As análises foram realizadas com e sem a presença dos casos extremos uni e multivariados nos estudos da prese nte pesquisa. Nas análises do Estudo 1, não foram detectadas diferenças nos resultados com e sem casos extremos e, por isso, optou-se por utilizar os arquivos com a presença dos casos extremos uni e multivariados. Entretanto, no Estudo 2, a retirada dos casos extremos multivariados mostrou-se necessária.

Para o Estudo 1, realizaram-se análises dos componentes principais (PC) e de fatoração dos eixos principais (PAF). Os critérios utilizados foram: valores próprios superiores a 1; análise da distribuição dos valores próprios (scree plot); porcentagem mínima de 3\% de variância explicada para cada fator a ser extraído; cargas fatoriais superiores a 0,30; análise dos índices de consistência interna dos itens (alfa de Cronbach) e interpretabilidade das soluções propostas. Para cumprir o objetivo do Estudo 2 foram realizadas análises de regressão múltipla padrão e stepwise. Nessa etapa, as médias das respostas dos participantes às escalas foram transformadas em variáveis dos modelos de regressão múltipla. Sendo assim, a seguir são apresentados os resultados desse estudo. 


\section{RESULTADOS}

O objetivo do Estudo 2 era analisar o relacionamento entre as variáveis do modelo geral proposto na Figura 1. Entretanto, uma vez que o pareamento das respostas dos participantes a todas as escalas resultou em um arquivo de dados com número insuficiente de casos para a testagem dos dados, não foi possível testar o modelo geral. Para otimizar o aproveitamento de casos, foram construídos dois modelos de análise de regressão múltipla com diferentes combinações de variáveis preditoras da variável-critério, Impacto do Treinamento no Trabalho. Foram utilizadas como variáveis preditoras e critério as médias das respostas dos participantes às escalas (impfg).

Variáveis antecedentes incluídas no Modelo 1: horas de estudo na Internet (estudo_i); horas de estudo fora da ilternet (estudo_e); média das respostas aos itens que compõem o fator 1 (Busca de ajuda interpessoal) do instrumento Estratégias de Aprendizagem (estf1); o fator 2 (Elaboração e aplicação prática) do instrumento Estratégias de Aprendizagem (estf2); o fator 3 (Repetição, organização e busca de ajuda no material) do instrumento Estratégias de Aprendizagem (estf3); fator geral de reação ao desempenho do tutor (tutorfg); o fator geral de falta de suporte à transferência (supfg); e elaboração de um plano de negócios (imp25). Modelo 2: média das respostas aos itens que compõem o fator geral de reação aos procedimentos instrucionais (procedfg); o fator de geral de falta de suporte à transferência (supfg); e elaboração de um plano de negócios (imp25). As demais variáveis relacionadas à freqüência de participação nos procedimentos via Web foram excluídas das análises de regressão múltipla por não manterem correlações bivariadas significativas com a variável-critério e para garantir parcimônia, reduzindo as chances de Erro do Tipo I. As variáveis relacionadas aos dados pessoais e profissionais foram excluídas das amostras em função de os dados anteriores da literatura indicarem que essas variáveis não são preditoras significativas da variável-critério, Impacto do Treinamento no Trabalho, e também para garantir a parcimônia dos modelos.

Foram realizadas análises de regressão múltipla padrão para o Modelo 1 em virtude do tamanho da amostra $(\mathrm{N}=194)$ e análises de regressão múltipla padrão e stepwise para o Modelo 2, pois o número de casos $(\mathrm{N}=356)$ era suficiente, conforme critérios de Tabachnick e Fidell (2000). As variáveis inseridas nos dois modelos foram submetidas a análises exploratórias, segundo os procedimentos propostos por Tabachnick e Fidell (2000). Não foi encontrada nenhuma variável que apresentasse quantidade superior a $5 \%$ de dados omissos e, por isso, optouse pelo tratamento pairwise. Foram identificados seis casos extremos multivariados no Modelo 1, e dois casos no Modelo 2, os quais foram excluídos das análises.

\section{Resultados do modelo 1}

No Modelo 1 , os casos $(\mathrm{N}=188)$ foram submetidos à análise de regressão múltipla padrão. Na Tabela 1, podem-se observar as correlações entre as variá veis, os coeficientes de regressão não-padronizados (B), os coeficientes de regressão padronizados (ß), a contribuição individual de cada variável ( $\mathrm{sr}^{2}$ ), as médias das variáveis (X), os desvios-padrão (DP), a constante, $\mathrm{o} \mathrm{R}^{2}, \mathrm{o} \mathrm{R}^{2}$ ajustado e o $\mathrm{R}$.

$\mathrm{O}$ coeficiente de regressão múltipla $\mathrm{R}$ foi significativamente diferente de zero, $\mathrm{F}(8,179)=$ $13,68,<0,001$, sendo considerados limites de confiança de $95 \%$ para os dois coeficientes de regressão que eram diferentes significativamente de zero. Apenas três variáveis antecedentes contribuíram 
significativamente para a explicação de impacto em profundidade, a saber: Suporte $(\beta=0,30)$; Estratégias de Aprendizagem - fator $2(\beta=0,27)$; e Elaborei o plano de negócios $\beta=-0,32)$. Agrupadas, as 8 variáveis iniciais explicam 38\% (35\% ajustado) da variabilidade de impacto do treinamento no trabalho percebido pelos participantes. As três variáveis que contribuem significativamente com a explicação da variávelcritério explicam, conjuntamente, cerca de $23 \%$ da variabilidade de impacto.

Tabela 1 - Regressão múltipla padrão para o Modelo 1

\begin{tabular}{|c|c|c|c|c|c|c|c|c|c|}
\hline Variáveis & $\begin{array}{l}\text { impfg } \\
\text { (VD) }\end{array}$ & $\begin{array}{l}\text { Estudo } \\
\text { _i }\end{array}$ & estudo_e & estf1 & estf2 & estf3 & tutorfg & supfg & imp25 \\
\hline Estudo_i & 0,15 & & & & & & & & \\
\hline Estudo_e & 0,21 & 0,06 & & & & & & & \\
\hline estf1 & 0,09 & 0,06 & 0,14 & & & & & & \\
\hline estf2 & 0,37 & 0,07 & 0,23 & 0,00 & & & & & \\
\hline estf3 & 0,36 & 0,03 & 0,42 & 0,30 & 0,39 & & & & \\
\hline Tutorfg & 0,15 & 0,00 & 0,04 & $-0,01$ & 0,20 & $-0,01$ & & & \\
\hline Supfg & 0,29 & 0,06 & 0,04 & $-0,04$ & $-0,03$ & 0,10 & 0,02 & & \\
\hline imp25 & $-0,38$ & $-0,17$ & $-0,21$ & $-0,13$ & $-0,10$ & $-0,26$ & $-0,03$ & 0,05 & \\
\hline$B$ & & 0,00 & 0,00 & 0,00 & $0,32 *$ & 0,10 & 0,12 & $0,27^{*}$ & $-1,22^{*}$ \\
\hline$\beta$ & & 0,05 & 0,00 & 0,02 & 0,27 & 0,14 & 0,08 & 0,30 & $-0,32$ \\
\hline$S r^{2}$ & & 0,00 & 0,00 & 0,00 & 0,06 & 0,00 & 0,00 & 0,08 & 0,09 \\
\hline$X$ & 7,49 & 4,35 & 3,40 & 1,81 & 8,51 & 5,27 & 8,93 & 6,28 & 1,26 \\
\hline DP & 1,68 & 2,72 & 3,46 & 1,96 & 1,41 & 2,26 & 1,13 & 1,87 & 0,44 \\
\hline \multicolumn{10}{|c|}{ Constante $=2,83 ; \mathrm{R}^{2}=0,38 ; \mathrm{R}^{2}$ (ajustado) $=0,35 ; \mathrm{R}=0,62$} \\
\hline
\end{tabular}

Em suma, relataram mais impacto do treinamento no trabalho aqueles participantes do IPGN que elaboraram um plano de negócios, avaliaram desfavoravelmente o suporte à transferência e disseram utilizar com maior frequiência as estratégias de elaboração e aplicação prática dos conteúdos do curso. 


\section{Resultados do Modelo 2}

No Modelo 2, os casos $(\mathrm{N}=354)$ foram submetidos à análise de regressão múltipla padrão. Como pode ser visto na Tabela 2, o coeficiente de regressão múltipla $\mathrm{R}$ foi significativamente diferente de zero, $\mathrm{F}(3,348)=38,53,<0,001$, sendo considerados limites de confiança de $95 \%$ para os dois coeficientes de regressão que eram diferentes significativamente de zero. As três variáveis antecedentes que contribuíram significativamente para a explicação de impacto do treinamento no trabalho foram: Suporte $(\beta=0,25)$; Satisfação com os procedimentos instrucionais $(\beta=0,32)$ e Elaborei o plano de negócios $\beta=-0,24)$. Agrupadas, as três variáveis antecedentes explicam 25\% (24\% ajustado) da variabilidade de impacto do treinamento no trabalho.

Tabela 2 - Regressão múltipla padrão para o Modelo 2

\begin{tabular}{|c|c|c|c|c|c|c|c|}
\hline Variáveis & $\begin{array}{l}\text { Proffg } \\
\text { (VD) }\end{array}$ & Procedfg & supfg & Imp25 & $\mathrm{B}$ & $\beta$ & $\mathrm{sr}^{2}$ \\
\hline Procedfg & 0,36 & & & & $0,47 *$ & 0,32 & 0,09 \\
\hline Supfg & 0,29 & 0,10 & & & $0,21 *$ & 0,25 & 0,06 \\
\hline Imp25 & $-0,27$ & $-0,07$ & $-0,03$ & & $-0,86^{*}$ & $-0,24$ & 0,06 \\
\hline$X$ & 7,60 & 8,09 & 6,31 & 1,29 & & & \\
\hline DP & 1,65 & 1,10 & 1,95 & 0,45 & & & \\
\hline \multicolumn{8}{|c|}{ Constante $=3,54 ; \mathrm{R}^{2}=0,25 ; \mathrm{R}^{2}$ (ajustado) $=0,24 ; \mathrm{R}=0,50}$. \\
\hline
\end{tabular}

$\mathrm{Na}$ análise de regressão stepwise, os resultados descritos na Tabela 2 foram, de modo geral, confirmados. Em suma, os participantes que relataram maior impacto do treinamento no trabalho foram aqueles que ficaram mais satisfeitos com os procedimentos instrucionais, perceberam maior falta de suporte à transferência e elaboraram um plano de negócios.

\section{DISCUSSÃO}

Todas as escalas construídas neste estudo mostraram se psicometricamente confiáveis e válidas. As escalas obtidas nesta pesquisa devem ser aplicadas em outras organizações, bem como em diferentes cursos com mídias diversificadas. Cursos semipresenciais ou totalmente a distância, utilizando mídias 
como Internet, CD-ROM, videoconferência, rádio e televisão e material impresso, devem ser avaliados à luz dos modelos testados neste estudo.

Os itens da Escala de Falta de Suporte à Transferência referem se a obstáculos que o indivíduo pode eventualmente enfrentar para manter o empreendimento aberto e lucrativo. $O$ papel da organização nesse caso seria o de oferecer apoio aos indivíduos e/ou cursos que promovam o desenvolvimento de estratégias de enfrentamento para essas situações.

No Modelo 1, a variável que explicou a maior parte da variabilidade de impacto do treinamento no trabalho foi elaboração de um plano de negócios (imp25). Por uma falha metodológica essa variável, que inicialmente fazia parte da Escala de Impacto do Treinamento no Trabalho, não foi colocada na mesma matriz de dados da análise fatorial da referida escala. Entretanto, novas análises foram feitas e o item não permaneceu na Escala de Impacto do Treinamento no Trabalho. Essa falha acabou chamando a atenção para o fato de que elaborar um plano de negócios é, na verdade, uma importante variável explicativa de impacto. Desse modo, deveria ser uma atividade obrigatória e necessariamente avaliada ao final do curso, o que não ocorreu no IPGN.

Neste estudo a variável relativa a suporte explicou uma porção significativa da variabilidade de impacto do treinamento no trabalho, corroborando, em parte, os achados de diversos autores. Entretanto, a porcentagem de explicação dessa variável sobre impacto, neste estudo, foi muito menor do que as obtidas nas pesquisas acima citadas. Isso se deve, provavelmente, à pequena ênfase dada ao apoio familiar no instrumento e ao fato de que as variáveis de suporte, usadas no presente estudo, são predominantemente referentes à gestão de um empreendimento já instalado, o que pode ter introduzido erro de medida, pois grande parte da amostra de participantes não é composta por empresários. Além disso, o curso IPGN é voltado predominantemente para o planejamento prévio de abertura de um negócio, e não para sua manutenção.

Os resultados deste trabalho, entretanto, diferem de todos os outros anteriormente citados no que se refere à direção do relacionamento entre suporte e impacto. Neste estudo, avaliações desfavoráveis de suporte à transferência é que estiveram relacionadas positivamente com impacto. Esse resultado não é surpreendente, uma vez que na primeira parte do curso IPGN é ensinado aos participantes que um empreendedor de sucesso é aquele que transforma obstáculos em desafios. Nesse sentido, aumentar a percepção de ambiente desfavorável aumentaria o mérito daquele indivíduo que o enfrenta como um desafio. O fenômeno da desejabilidade social pode ter, nesse caso, interferido nas respostas dos participantes, fazendo-os avaliar favoravelmente seu próprio comportamento e desfavoravelmente o ambiente.

Entre as variáveis relativas a características da clientela, Estratégia de Aprendizagem foi a única que explicou o impacto do treinamento no trabalho. Entretanto, apenas Elaboração e Aplicação Prática do Conteúdo, que inclui estratégias cognitivas e comportamentais, contribuiu significativamente na explicação de impacto do treinamento no trabalho. Não foi encontrado nenhum estudo, na revisão de literatura feita na presente pesquisa, que analisasse o relacionamento entre estratégias de aprendizagem e impacto do treinamento no trabalho. As pesquisas analisadas estudam estratégias de aprendizagem como preditoras de aprendizagem (Warr e Bunce, 1995, p. 347; Warr e Downing, 2000, p. 311) e reação (Warr e Bunce, 1995, p. 347). Warr e Bunce (1995, p. 347) estudaram o relacionamento entre estratégias de aprendizagem e comportamento no cargo, porém não encontraram relacionamento direto significativo entre essas variáveis. O fato de Busca de Ajuda Interpessoal não ter entrado como variável preditora de impacto do treinamento no trabalho pode ter ocorrido em função da baixa frequiência de 
uso dos recursos instrucionais de interação oferecidos pelo curso com o intuito de desenvolver um ambiente colaborativo de aprendizagem por parte dos participantes. O fato de Repetição, Organização e Ajuda do Material não terem entrado como preditores da variável-critério deve-se, provavelmente, à ausência de atribuições de notas às avaliações de aprendizagem. Os itens incluídos nessa escala provavelmente são mais utilizados quando as atividades a serem realizadas necessitam de retenção de informações.

A variável Reação ao Tutor não contribuiu significativamente na explicação de impacto do treinamento no trabalho. Este fato pode ser explicado pela baixa freqüência de participação dos indivíduos no recurso "tira-dúvidas" oferecido pelo curso para incentivar a interação tutor/aluno. Esse resultado não é surpreendente quando se observa a proporção de alunos por tutor (200 para 1), o que pode ter dificultado o contato personalizado do tutor com os aprendizes.

As variáveis referentes à quantidade de horas de estudo dentro e fora da Internet também não contribuíram com a explicação de impacto do treinamento no trabalho, provavelmente pelo fato de os participantes terem dedicado bem menos tempo aos estudos dos módulos do curso do que o sugerido pela organização.

No Modelo 2, a variável antecedente que explicou a maior parte da variabilidade de impacto do treinamento no trabalho, medido em profundidade, foi Reação aos Procedimentos, ou seja, alunos mais satisfeitos com os procedimentos do curso são também os que relatam maior aplicação de conhecimento adquirido no trabalho. Esse resultado corrobora os achados de Abbad (1999) e Sallorenzo (2000). Falta de Suporte à Transferência foi a segunda maior preditora da variável-critério estudada, corroborando, em parte, os resultados de pesquisa mencionados na discussão sobre o Modelo 1. Entretanto, tal como na discussão do Modelo 1, a porcentagem de explicação dessa variável sobre impacto foi menor do que aquelas obtidas nas pesquisas anteriormente citadas. Isso ocorreu pelos mesmos motivos descritos na discussão do Modelo 1. Novamente a variável Elaboração de um Plano de Negócio contribuiu para a explicação da ocorrência de impacto do treinamento no trabalho. Desse modo, deveria ser uma atividade obrigatória e necessariamente avaliada ao final do curso.

\section{CONSIDERAÇÕES FINAIS}

Os baixos coeficientes de determinação dos modelos de regressão múltipla indicam a existência de outras variáveis, não abordadas neste estudo, que explicam grande parcela da variabilidade de impacto do treinamento no trabalho. Apesar dos baixos coeficientes, os modelos reduzidos adaptados de treinamentos presenciais mostram-se adequados para a avaliação de treinamentos a distância, já que, como discutido anteriormente, essa linha de pesquisa apresenta lacunas teóricas e metodológicas quanto às avaliações sistemáticas da eficácia de tais programas instrucionais. Entretanto, em estudos futuros, será necessária a inclusão de novas variáveis explicativas no modelo de avaliação de impacto, como aprendizagem, motivação, valor instrumental do treinamento e ambiente eletrônico do curso, entre outras ${ }^{1}$. 
Em cursos a distância, variáveis referentes a "características da clientela" podem assumir maior relevância na explicação de impacto. Isso porque se sabe que o estudante de cursos a distância precisa ter em seu repertório competências complexas de autogestão da aprendizagem, as quais, provavelmente, são fortemente influenciadas por disposições individuais como locus de controle, autoeficácia e, principalmente, variáveis motivacionais. O "valor instrumental do treinamento" para o indivíduo talvez seja uma das mais importantes variáveis antecedentes de resultados de cursos abertos e gratuitos como o IPGN, já que esse conceito se refere à escolhas particulares do indivíduo e aos benefícios que o treinamento pode oferecer para o próprio participante relacionados a resultados futuros (Lacerda e Abbad, 2003, p. 77).

Nos modelos de explicação de impacto do treinamento no trabalho, faltou a variável Aprendizagem. Em pesquisas futuras, seria necessária a mensuração dos níveis de aprendizagem dos participantes por meio de pré e pós-testes.

As contribuições mais valiosas deste estudo consistem, pois, na construção e validação de cinco novas escalas de avaliação de treinamentos a distância que possibilitaram o teste dos modelos reduzidos de avaliação de treinamento. Além disso, neste estudo foi possível realizar validações estatísticas da Escala de Impacto do Treinamento no Trabalho, medidas em profundidade, fato raro na área, segundo Borges-Andrade (2002, p. 31). A revisão de literatura da área de avaliação de treinamento e de estudos sobre estratégias de aprendizagem também foi uma importante contribuição para o estudo, bem como o desenvolvimento de estratégias de coleta de dados pela Internet.

Os objetivos da pesquisa foram atingidos, algumas hipóteses foram confirmadas e outras refutadas: 1) determinadas estratégias de aprendizagem cognitivas e comportamentais contribuíram na explicação de impacto, entretanto os participantes não usaram estratégias diferentes das utilizadas em cursos tradicionais como se esperava; 2) a satisfação com o treinamento contribuiu com a explicação de impacto, porém a magnitude da contribuição foi menor do que a encontrada em modelos de avaliação de cursos presenciais; 3) a falta de suporte à transferência influenciou positivamente o impacto do treinamento no trabalho, o que vai de encontro à direção do relacionamento encontrada em resultados de outras pesquisas analisados na revisão de literatura. Variáveis de suporte, também neste estudo, continuam figurando entre as variáveis que mais explicam impacto de treinamento no trabalho; 4) as variáveis referentes a características da clientela, satisfação com o treinamento e falta de suporte à transferência explicaram, em conjunto, uma porção razoável da variabilidade de Impacto do Treinamento no Trabalho.

Entre as limitações desta pesquisa estão: 1) enorme perda de dados ocasionada por inúmeras falhas de programação dos questionários e quedas de conexão dos computadores dos participantes com a Internet durante o preenchimento das escalas; 2) baixo índice de devolução dos questionários, principalmente daqueles aplicados após o curso; 3) inexistência de pré-testes de conhecimentos; 4) falta de informações válidas sobre aprendizagem; e 5) não-realização de análises comparativas dos perfis de participantes concluintes e não-concluintes do curso IPGN. Essas falhas ou lacunas da pesquisa impossibilitaram o teste do modelo completo proposto e dificultaram a avaliação do quanto o curso foi capaz de desenvolver as competências descritas nos objetivos, assim como dificultaram a avaliação da generalidade dos resultados.

Diante do exposto, sugere-se a realização de mais análises, utilizando os dados obtidos nesta pesquisa, entre as quais: 1) análise do relacionamento entre variáveis de Reação aos Procedimentos e ao Tutor com os índices de evasão e de frequiência de participação nos recursos eletrônicos oferecidos 
no curso; 2) análise do relacionamento entre as variáveis de Estratégias de Aprendizagem com índices de evasão.

Sugere-se também a realização de mais pesquisas para: 1) examinar o relacionamento entre os três mais tradicionais níveis de avaliação - Reações, Aprendizagem e Impacto de treinamentos a distância no trabalho; 2) analisar o relacionamento entre Características da Clientela e de Suporte à Transferência com os níveis de Reação, Aprendizagem e Impacto; 3) Avaliar quais dessas variáveis explicam evasão em treinamentos a distância $(\mathrm{TaD})$; 4) avaliar quais variáveis explicam retenção e generalização de competências em $\mathrm{TaD}$; 5) verificar a necessidade de adaptar os instrumentos de avaliação propostos neste estudo, buscando inserir variáveis de resultados de treinamentos a distância, provenientes de outras disciplinas ou áreas de estudo que trabalham com tecnologias da informação e da comunicação, tais como computação, ergonomia, administração e engenharia de produção; 6) revalidar os instrumentos de valor instrumental, auto-eficácia e locus controle, mencionados anteriormente, utilizando dados de participantes de $\mathrm{TaD}$ ); 7) revisar os conceitos de impacto e aprendizagem à luz da abordagem multinível de Kozlowski, Brown, Weissbein, Cannon-Bowers e Salas, (2000, p. 157), conforme sugerido por Abbad, Pantoja e Pilati (2003, p. 181).

\section{NOTAS}

${ }^{1}$ Este estudo é parte de uma pesquisa mais ampla que envolve a avaliação do curso nos níveis de satisfação, aprendizagem e impacto do treinamento no trabalho. Assim, em um primeiro momento de coleta, ao final do curso, foram obtidos dados de: estratégias de aprendizagem; reação aos procedimentos instrucionais, reação ao desempenho do tutor, à interface gráfica, a resultados e aplicabilidade; aprendizagem; e dados pessoais dos alunos. No segundo momento, aqui enfocado, foram coletados dados de impacto em profundidade e amplitude e falta de suporte à transferência. Detalhes da pesquisa estão disponíveis nas obras de Carvalho (2003) e Zerbini (2003).

\section{REFERÊNCIAS BIBLIOGRÁFICAS}

ABBAD, G. Um modelo integrado de avaliação de Impacto do Treinamento no Trabalho - IMPACT. Brasília, 1999. Tese (Doutorado) - Instituto de Psicologia da Universidade de Brasília.

ABBAD, G., PILATI, R.; PANTOJA, M. J. Avaliação de treinamento: análise da literatura e agenda de pesquisa. Revista de Administração-USP, v. 38, n. 3, p. 181-91, 2003. 
ABBAD, G.; GAMA, A. L. G.; BORGES -ANDRADE, J. E. Treinamento: Análise do Relacionamento da Avaliação nos Níveis de Reação, Aprendizagem e Impacto no Trabalho. Revista de Administração Contemporânea, v. 4, n. 3, p. 25-45, 2000.

BORGES-ANDRADE, J. E. Avaliação Somativa de Sistemas Instrucionais: integração de três propostas. Tecnologia Educacional, v. 11, n. 46, p. 29-39, 1982.

BORGES-ANDRADE, J. E. Desenvolvimento de Medidas em Avaliação de Treinamento. Estudos de Psicologia, v. 7 (Número Especial), p. 31-43, 2002.

BORGES-ANDRADE, J. E.; ABBAD, G. Treinamento no Brasil: reflexões sobre suas pesquisas. Revista de Administração, v. 31, n. 2, p. 112-25, 1996.

CARVALHO, R. Avaliação de treinamento a distância: reação, suporte à transferência e impacto do treinamento no trabalho. Dissertação (Mestrado) - Instituto de Psicologia da Universidade de Brasília, Brasília, 2003.

CHEUNG, D. Developing a student evaluation instrument for distance teaching. Distance Education, v. 19, n. 1, p. 23-34, 1998.

DEAN, A.; WEBSTER, L. Simulations in distance education-progress towards an evaluation instrument. Distance Education, v. 21, n. 2, p. 344-60, 2000.

HAMBLIN, A. C. Avaliação e Controle de Treinamento. São Paulo, McGraw-Hill do Brasil, 1978.

LACERDA, E. R. M.; ABBAD, G. Impacto do Treinamento no Trabalho: investigando variáveis motivacionais e organizacionais como suas preditoras. Revista de Administração Contemporânea, v. 7, n. 4, p. 77-96, 2003.

KIRKPATRICK, D. L. Evaluation of Training. In: Craig, R. L. Training and development handbook. New York: McGraw-Hill, p. 18.1-18.27, 1976.

KOZLOWSKI, S. W. J.; BROWN, K. G.; WEISSBEIN, D.A; CANNON-BOWERS, J. A; SALAS, E. In: KLEIN, K. J.; KOSLOWSKI, S. W. J. (eds.). Multilevel Theory, Research, and Methods in Organizations: Foundations, Extensions and New Directions. San Francisco: Jossey-Bass, 2000. p. 157-210.

ROULLIER, J. Z.; GOLDSTEIN, I. L. The relationship between organizational transfer climate and positive transfer of training. Human Resource Development Quarterly, v. 4, n. 4, p. 377-90, 1993.

SALAS, E.; CANNON-BOWERS, J. The Science of Training: a decade of progress. Annual Review of Psychology, v. 52, p. 471-99, 2001.

SALLORENZO, L. H. Avaliação de Impacto do Treinamento no Trabalho: analisando e comparando modelos de predição. Dissertação (Mestrado) - Instituto de Psicologia da Universidade de Brasília, Brasília, 2000.

TABACHNICK, B. G.; FIDELL, L. S. Using Multivariate Statistics. New York: Harper \& Row, 1996. 


\section{IMPACTO DE TREINAMENTO NO TRABALHO VIA INTERNET \\ Thaís Zerbini - Gardênia Abbad}

WALKER, S. The value of building skills with online technology: online training costs and evaluation at the Texas Natural Resource Conservation Commission. In: SCHEIBER, D. A.; BERGE, Z. L. (orgs.). Distance Training: how innovative organizations are using technology to maximize learning and meet business objectives . San Francisco: Jossey-Bass Publishers, 1998. p. 270-86.

WARR, P.; ALLAN, C. Learning strategies and occupational training. Internacional Review of Industrial and Organizational Psychology, v. 13, p. 83-121, 1998.

WARR, P.; BUNCE, D. Trainee Characteristics and Outcomes of Open Learning. Personnel Psychology, v. 48, p. 347-75, 1995.

WARR, P.; DOWNING, J. Learning strategies, learning anxiety and knowledge acquisition. British Journal Psychology, v. 91, p. 311-33, 2000.

ZERBINI, T. Estratégias de Aprendizagem, Reações de um Curso via Internet, Reações ao Tutor e Impacto do Treinamento no Trabalho, 2003. Dissertação (Mestrado) - Instituto de Psicologia, Universidade de Brasília, Brasília.

\section{Artigo recebido em 05.10.2004. Aprovado e m 10.03.2005.}

\section{Thaís Zerbini}

Professora na Faculdade Millenniun de Brasília (DF). Doutoranda em Psicologia na UnB.

Interesses de pesquisa nas áreas de avaliação de impacto de treinamentos nos níveis do indivíduo e da organização, eficácia de ações de educação corporativa e educação profissional à distância e eficácia de planejamento instrucional de sistemas educacionais presenciais e à distância.

E-mail: zerbini@unb.br

Endereço: SQN 410 Bloco E, ap. 105, Asa Norte, Brasília - DF, 70865-050

\section{Gardênia Abba d}

Professora Adjunta do Departamento de Psicologia Social e do Trabalho da UnB. Doutora em psicologia pela UnB.

Interesses de pesquisa nas áreas de avaliação de impacto de treinamentos nos níveis do indivíduo e da organização, eficácia de ações de educação corporativa e educação profissional e corporativa à distância.

E-mail: gardenia@unb.br

Endereço: SQN 205 Bloco C, ap. 201, Asa Norte, Brasília - DF, 70865-050 